\title{
Contribution of MMP2 Promoter Genotypes to Oral Cancer Susceptibility, Recurrence and Metastasis in Taiwan
}

\author{
CHIA-WEN TSAI ${ }^{1 *}$, HUAI-MEI HSU ${ }^{1,2 *}$, YUN-CHI WANG $^{1 *}$, WEN-SHIN CHANG $^{1,2}$, LIANG-CHUN SHIH $^{3}$, \\ KUO-TING SUN ${ }^{4}$, YI-WEN HUNG ${ }^{5}$, YI-CHIN YANG ${ }^{5}$, CHI-LI GONG ${ }^{6}$ and DA-TIAN BAU ${ }^{1,2,7}$ \\ ${ }^{1}$ Terry Fox Cancer Research Laboratory, Translational Medicine Research Center, \\ ${ }^{3}$ Department of Otolaryngology, and ${ }^{4}$ Division of Pediatric Dentistry, \\ China Medical University Hospital, Taichung, Taiwan, R.O.C.; \\ ${ }^{2}$ Graduate Institute of Biomedical Sciences and ${ }^{6}$ Department of Physiology, \\ China Medical University, Taichung, Taiwan, R.O.C.; \\ ${ }^{5}$ Department of Medical Research, Taichung Veterans General Hospital, Taichung, Taiwan, R.O.C.; \\ ${ }^{7}$ Department of Bioinformatics and Medical Engineering, Asia University, Taichung, Taiwan, R.O.C.
}

\begin{abstract}
Aim: Metalloproteinase 2 (MMP2) is a multifunctional protein which has been shown to be up-regulated in patients with oral cancer, especially those with lymph node metastasis. However, the association of MMP2 genotype with oral cancer risk or metastatic behavior is unknown. This study aimed to evaluate the role of MMP2 promoter 1306 and -735 genotypes in the risk of oral cancer and metastasis. Materials and Methods: In this case-control study, MMP2 promoter 1306 (rs243865) and -735 (rs2285053) genotypes and their interaction with consumption of areca, cigarettes, and alcohol in determining oral cancer risk were investigated among 788 patients with oral cancer and 956 gender-matched healthy controls. In addition, their role in oral cancer metastasis were also examined. Results: The distribution of CC, CT and TT for MMP2 promoter 1306 genotype was 79.0, 20.1 and 0.9\% in the oral cancer group and 68.7, 29.2 and 2.1\% in the noncancer control group, respectively ( $p$ for trend=4.3E-6). The allelic frequency distributions showed that the variant $T$ allele of MMP2 promoter 1306 conferred lower oral cancer susceptibility than the wild-type $C$ allele (odds ratio $=0.61$, $95 \%$ confidence interval $=0.50-0.75, p=1.1 E-6)$. As for the MMP2 -735 genotypes, there was no differential distribution
\end{abstract}

\footnotetext{
*These Authors contributed equally to this study.

Correspondence to: Da-Tian Bau, Chi-Li Gong, Terry Fox Cancer Research Laboratory, Translational Medicine Research Center, China Medical University Hospital, 2 Yuh-Der Road, Taichung, 404 Taiwan, R.O.C. Tel: +886 422053366 (Ext. 5805), e-mail: datian@mail.cmuh.org.tw; artbau2@gmail.com
}

Key Words: Genotype, metastasis, MMP2, oral cancer, polymorphism, Taiwan. in genotypic or allelic frequencies. The variant $C T$ and $T T$ genotypes were also associated with lower metastasis rates within 5 years among the patients with oral cancer (odds ratio $=0.34,95 \%$ confidence interval $=0.15-0.80, p=0.0102)$. Conclusion: The CT and TT genotypes of MMP2 promoter 1306 may have a protective effect on oral cancer susceptibility and metastasis risk within 5 years for Taiwanese. They may serve as predictive markers for oral cancer in precise medical practice.

From the viewpoint of epidemiology, oral cancer is the tenth most commonly diagnosed cancer worldwide, with the highest incidence density in Taiwan (1). According to the updated annual report from Taiwan government, oral cancer is the fourth cause of cancer-related death among males in Taiwan and fifth among all citizens (2). Unluckily, patients with oral cancer in Taiwan and all over the world suffer from the threat of recurrence and metastasis. Those at higher risk of oral cancer recurrence or metastasis should be detected earlier and followed-up more frequently to enjoy longer life, with the development of useful markers for prognosis prediction. Although several predictive biomarkers for oral cancer in Taiwan have been revealed (3-9), genomic biomarkers of oral cancer risk, especially those useful for prediction of recurrence/metastasis are of great interest. Among them, the practical biomarkers for oral cancer metastasis are urgently in need.

Extracellular matrix (ECM) structures play an important role in micro-environmental remodeling during tumorigenesis (10). The matrix metalloproteinases (MMPs) are a family of enzymes involved in ECM remodeling via controlling the degradation of ECM components, such as those in connective tissue matrices $(10,11)$. In literature, MMPs were reported to be related to the regulation of oral cancer invasion and metastasis (12). 
Table I. Characteristics of the 788 patients with oral cancer and 956 controls investigated.

\begin{tabular}{lccc}
\hline Characteristic & $\begin{array}{c}\text { Controls } \\
(\mathrm{n}=956)\end{array}$ & $\begin{array}{c}\text { Cases } \\
(\mathrm{n}=788)\end{array}$ & $p$-Value \\
\hline Age (years) & & & \\
$\quad$ Mean (SD) & $56.6(8.7)$ & $55.8(9.9)$ & 0.7951 \\
Gender, n (\%) & & & \\
Male & $727(76.0 \%)$ & $599(76.0 \%)$ & $>0.99$ \\
Female & $229(24.0 \%)$ & $189(24.0 \%)$ & \\
Personal habits, n (\%) & & & \\
Areca chewing & $506(52.9 \%)$ & $661(83.9 \%)$ & $<\mathbf{0 . 0 0 0 1}$ \\
Cigarette smoking & $667(69.8 \%)$ & $595(75.5 \%)$ & $\mathbf{0 . 0 0 8 4}$ \\
Alcohol drinking & $641(67.1 \%)$ & $560(71.1 \%)$ & 0.0773 \\
Primary tumor site, $\mathrm{n}(\%)$ & & & \\
Tongue & & $325(41.2 \%)$ & \\
Buccal mucosa & & $294(37.3 \%)$ & \\
Mouth floor & & $30(3.8 \%)$ & \\
Retromolar trigone & & $26(3.3 \%)$ & \\
Alveolar ridge & & $18(2.3 \%)$ & \\
Palate & & $18(2.3 \%)$ & \\
Lip & & $39(4.9 \%)$ & \\
Other & & $38(4.9 \%)$ & \\
\hline
\end{tabular}

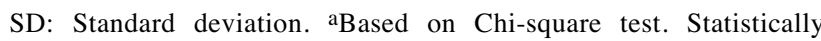
significant values are shown in bold.

In recent years, the role of MMPs in the process of tumor invasion has received continuous attention and it was reported that MMP2 played an important role in the degradation of extracellular matrix mediated by glioma cells (13). MMP2 gene is located on chromosome 16q21 and composed of 12 introns and 13 exons (14). Promoter 1306 (rs243865) and -735 (rs2285053) single nucleotide polymorphisms (SNP) of $M M P 2$, together with those of its inhibitor TIMP2, can affect their protein or mRNA expression and tumor invasion by altering the transcriptional activity of its own genes, eventually involving in the development of several types of cancer, including breast, lung, esophageal and colon cancer (15-18). MMP2 was reported to be up-regulated in patients with oral squamous cell carcinoma, especially those with lymph node metastasis (19). Thus, in the present work, a case-control genotyping study was performed to investigate the correlations of MMP2 promoter 1306 (rs243865) and -735 (rs2285053) polymorphisms with the susceptibility and metastatic prognosis of oral cancer in Taiwan.

\section{Materials and Methods}

Oral cancer patient and control group collection. The current study was approved by the Institutional Review Board (DMR101-IRB1306) of our Hospital. Firstly, 788 patients diagnosed with oral cancer voluntarily provided $5 \mathrm{ml}$ of their peripheral blood and completed a self-administered questionnaire. Then, a total of 956 non-cancer healthy individuals as controls were selected by matching for age and gender after initial random sampling from the Health Examination Cohort of the hospital, and they also contributed their blood and completed the questionnaire. The questionnaire administered to each participant included questions on medical history and their individual frequency of alcohol consumption, areca chewing and smoking habit. Self-reported alcohol consumption, areca chewing and smoking habits were evaluated and classified as categorical variables. Information on these factors obtained as more than twice a week for years was recorded as "ever". The male versus female ratio was $76 \%$ to $24 \%$ in each group, perfectly matched with each other. The recurrence and metastasis status of each patient were closely followed at least twice per year after their surgery. The mean age of the patients and the controls was $55.8(\mathrm{SD}=9.9)$ and $56.6(\mathrm{SD}=8.7)$ years, showing that the matching was successful, causing a non-significantly differential distribution between the case and control groups. More detailed information is summarized in Table I.

Genotyping processes. The genomic DNA from the peripheral blood leukocytes donated by each participant was prepared within $24 \mathrm{~h}$ after collection applying the QIAamp Blood Mini Kit (Blossom, Taipei, Taiwan, ROC), and stored at $-80^{\circ} \mathrm{C}$ until processed as per our previous articles (3-5). In this study, the genotypes at -1306 and -735 polymorphic sites in the $M M P 2$ promoter region were determined for all the individuals in both the control and oral cancer groups. In brief, the polymorphic sites were genotyped by typical polymerase chain reaction-restriction fragment length polymorphism (PCR-RFLP) methodologies using a BioRad Mycycler (BioRad, Hercules, CA, USA). Each PCR reaction consisted of an initial cycle at $94^{\circ} \mathrm{C}$ for $5 \mathrm{~min} ; 40$ cycles of $94^{\circ} \mathrm{C}$ for $30 \mathrm{~s}, 55^{\circ} \mathrm{C}$ for $30 \mathrm{~s}$, and $72^{\circ} \mathrm{C}$ for $30 \mathrm{~s}$; and a final extension at $72^{\circ} \mathrm{C}$ for $10 \mathrm{~min}$. After PCR, the SNP-containing DNA amplicons were subjected to individual overnight digestion by restriction endonucleases. Following digestion, each sample was immediately analyzed by agarose gel electrophoresis. All the genotypic processing was repeated by two researchers independently, and blindly, and the results were $100 \%$ concordant. The details of primer sequences and the restriction enzymes are provided in Table II.

Statistical analysis. The Student's $t$-test was used for comparing the distribution of ages between the two groups. Pearson's chi-square test was applied to compare the distribution of the MMP2 -1306 and -735 genotypes among the subgroups, and also to examine the possible interaction among the indices of interest. The associations between the MMP2 -1306 and -735 genotypes and oral cancer risk were estimated by computing odds ratios (ORs) and their 95\% confidence intervals (CIs) from logistic regression analysis. Any difference with an outcome of $p<0.05$ was considered statistically significant.

\section{Results}

The frequency distributions of selected demographic characteristics including age, gender, personal habits and primary tumor sites for the 788 patients with oral cancer and 956 non-cancer controls are summarized in Table I. Since we applied frequency matching for age and gender to recruit the non-cancer healthy controls, there was no difference in the distributions of age and gender between the control and case 
Table II. Summary of the primers, restriction enzymes and amplicon size after enzyme cutting for matrix metalloproteinase-2 (MMP2) genotyping polymerase chain reaction-restriction fragment length polymorphism conditions.

\begin{tabular}{lccc}
\hline Polymorphic site & Primer sequences & Restriction enzyme & Amplicon size after cutting, bp \\
\hline MMP2 -1306 & Forward 5'-CTTCCTAGGCTGGTCCTTACTGA-3' & XspI & C: $188+5$ \\
& Reverse 5'-CTGAGACCTGAAGAGCTAAAGAGCT-3' & & T: $162+26+5$ \\
MMP2 -735 & Forward 5'-GGATTCTTGGCTTGGCGCAGGA-3' & HinfI & C: 391 T: $338+53$ \\
& Reverse 5'-GGGGGCTGGGTAAAATGAGGCTG-3' & & T \\
\hline
\end{tabular}

groups (Table I). For these investigated individuals, betel quid chewers and smokers were found at higher percentages in patients with oral cancer than in the controls (Table I). The most frequently diagnosed primary tumors occurred in the tongue $(41.2 \%)$ and buccal mucosa $(37.3 \%)$ for patients with oral cancer in Taiwan.

The distributions of the MMP2 promoter 1306 and -735 genotypes among the non-cancer controls and the patients with oral cancer are presented and statistically analyzed in Table II.

The genotypes of $M M P 2$ promoter 1306 were differently distributed between oral cancer and non-cancer control groups ( $\mathrm{p}$ for trend $=4.3 \times 10^{-6}$ ) (Table III, top). In detail, the $M M P 2$ promoter 1306 heterozygous CT and homozygous TT were both associated with reduced oral cancer risk ( $p=0.0001$ and 0.0192 , respectively; Table III). On the contrary, the genotypes of $M M P 2$ promoter 735 were not differently distributed between oral cancer and non-cancer control groups ( $p$ for trend=0.8932) (Table III).

To confirm the findings in Table III, the analysis of allelic frequency distribution for MMP2 promoter 1306 and -735 was also conducted and the results are summarized in Table IV. Supporting the findings that heterozygous CT and homozygous TT genotypes of MMP2 promoter 1306 were associated with oral cancer risk, the variant $\mathrm{T}$ allele was found at $10.9 \%$ in the case group, significantly lower than that of $16.7 \%$ in the control group $\left(p=1.1 \times 10^{-6}\right)$. To sum up, there was a significant difference in the allelic frequencies of $M M P 2$ promoter 1306 between the control and oral cancer groups (Table IV). It was also validated that there was no significant differential distribution $(p=0.6604)$ for the allelic frequencies of $M M P 2$ promoter -735 (Table IV).

Next, we were interested whether the $M M P 2$ promoter 1306 and -735 genotypes could serve as a predictor for the prognosis of patients with oral cancer. To fulfill this, the distributions of the MMP2 promoter 1306 and -735 genotypes were examined among the patients stratified by disease recurrence and metastasis status with a cut-off of 5 years. Firstly, the patients with oral cancer carrying the genotype of CT or TT at MMP2 promoter 1306 were at lower risk of metastasis within 5 years of surgery $(p=0.0102)$ than those patients carrying the wild-type $\mathrm{CC}$ genotype at MMP2 promoter 1306 (Table V). On the contrary, there was no differential distribution of the $M M P 2$ promoter 1306 genotype between patients with and those without recurrence within 5 years (Table V). There was no positive evidence for the involvement of $M M P 2$ promoter 735 genotype in determining the recurrence or metastasis status for these Taiwanese patients with oral cancer (data not shown).

\section{Discussion}

In the current case-control association study, the contribution of MMP2 promoter 1306 and -735 genotypes to oral cancer risk was firstly evaluated among Taiwanese, where the male oral cancer density is highest in the world. The two SNP loci, -1306 and -735 , are both located upstream of the MMP2 transcriptional start site. Their variation might destroy the binding site of SP1, resulting in reduction of gene transcription, and eventually reduce the expression of MMP2 (20). The results showed that both the genotypic and the allelic frequencies of $M M P 2$ promoter 1306 were differentially distributed between the 788 patients with oral cancer and 956 non-cancer healthy controls (Tables III and IV). In addition, the variant T-bearing genotypes at MMP2 promoter 1306 were associated with reduced risk of cancer metastasis in addition to cancer susceptibility itself (Table V).

MMP2 protein, also called gelatinase, is involved in the degradation of the intact fibrillar collagen, elastin, endothelin, fibroblast growth factor, MMP9, MMP13, plasminogen, and transforming growth factor $\beta$ (21). MMP2 has been reported to play an important role in ECM degradation, which is important for primary tumor cells to undergo invasion and migration $(22,23)$. Mounting evidence indicates that activated MMP2 is observed and linked with poor prognosis of many types of cancer including melanoma, colorectal, breast, ovarian, lung and prostate cancer, reviewed and summarized in (24). MMP2 is thought to promote epithelial-mesenchymal transition through the degradation of type IV collagen, the most abundant component of the basement membrane. The basement membrane is important for maintaining tissue organization 
Table III. Distribution of matrix metalloproteinase-2 (MMP2) genotypes among the patients with oral cancer and non-cancer controls.

\begin{tabular}{|c|c|c|c|c|c|c|}
\hline & \multicolumn{2}{|c|}{ Controls } & \multicolumn{2}{|c|}{ Patients } & \multirow[t]{2}{*}{ OR $(95 \% \mathrm{CI})$} & \multirow[t]{2}{*}{$p$-Value ${ }^{a}$} \\
\hline & $\mathrm{n}$ & $\%$ & $\mathrm{n}$ & $\%$ & & \\
\hline \multicolumn{7}{|c|}{$M M P 2-1306$} \\
\hline $\mathrm{CC}$ & 657 & $68.7 \%$ & 623 & $79.0 \%$ & 1.00 (Reference) & \\
\hline CT & 279 & $29.2 \%$ & 158 & $20.1 \%$ & $0.60(0.48-0.75)$ & 0.0001 \\
\hline $\mathrm{TT}$ & 20 & $2.1 \%$ & 7 & $0.9 \%$ & $0.37(0.16-0.88)$ & 0.0192 \\
\hline \multicolumn{7}{|c|}{$\begin{array}{l}P_{\text {trend }} \\
M M P 2-735\end{array}$} \\
\hline $\mathrm{CC}$ & 632 & $66.1 \%$ & 515 & $65.4 \%$ & 1.00 (Reference) & \\
\hline $\mathrm{CT}$ & 282 & $29.5 \%$ & 235 & $29.8 \%$ & $1.02(0.83-1.26)$ & 0.8333 \\
\hline $\mathrm{TT}$ & 42 & $4.4 \%$ & 38 & $4.8 \%$ & $1.11(0.71-1.75)$ & 0.6513 \\
\hline$P_{\text {trend }}$ & & & & & & 0.8932 \\
\hline
\end{tabular}

aBased on Chi-square test without Yates' correction. Statistically significant values are shown in bold.

Table IV. Distribution of allelic frequencies for matrix metalloproteinase-2 (MMP2) among patients with oral cancer and non-cancer controls.

\begin{tabular}{|c|c|c|c|c|c|c|}
\hline & \multicolumn{2}{|c|}{ Controls } & \multicolumn{2}{|c|}{ Patients } & \multirow[t]{2}{*}{ OR $(95 \% \mathrm{CI})$} & \multirow[t]{2}{*}{$p$-Value ${ }^{\mathrm{a}}$} \\
\hline & $\mathrm{n}$ & $\%$ & $\mathrm{n}$ & $\%$ & & \\
\hline \multicolumn{7}{|c|}{$M M P 2-1306$} \\
\hline $\mathrm{C}$ & 1,593 & $83.3 \%$ & 1,404 & $89.1 \%$ & 1.00 (Reference) & \\
\hline $\mathrm{T}$ & 319 & $16.7 \%$ & 172 & $10.9 \%$ & $0.61(0.50-0.75)$ & $1.1 \times 10^{-6}$ \\
\hline \multicolumn{7}{|c|}{$M M P 2-735$} \\
\hline $\mathrm{C}$ & 1,546 & $80.9 \%$ & 1,265 & $80.3 \%$ & 1.00 (Reference) & \\
\hline $\mathrm{T}$ & 366 & $19.1 \%$ & 311 & $19.7 \%$ & $1.04(0.88-1.23)$ & 0.6604 \\
\hline
\end{tabular}

'Based on chi-square test without Yates' correction. Statistically significant values are shown in bold.

Table V. Association of matrix metalloproteinase-2 (MMP2) genotypes with cancer recurrence and metastasis status.

\begin{tabular}{|c|c|c|c|c|}
\hline \multirow[t]{2}{*}{ Patient status } & \multicolumn{2}{|c|}{$M M P 2 \mathrm{C}-1306 \mathrm{~T}$ genotype } & \multirow[b]{2}{*}{ OR $(95 \% \mathrm{CI})$} & \multirow[b]{2}{*}{$p$-Value ${ }^{a}$} \\
\hline & $\mathrm{CC}$ & $\mathrm{CT}+\mathrm{TT}$ & & \\
\hline \multicolumn{5}{|l|}{ Recurrence status } \\
\hline No recurrence $>5$ years & 573 & 147 & 1.00 (Reference) & \\
\hline Recurrence $\leq 5$ years & 50 & 18 & $1.40(0.79-2.48)$ & 0.2409 \\
\hline \multicolumn{5}{|l|}{ Metastasis status } \\
\hline No metastasis $>5$ years & 561 & 159 & 1.00 (Reference) & \\
\hline Metastasis $\leq 5$ years & 62 & 6 & $0.34(0.15-0.80)$ & 0.0102 \\
\hline
\end{tabular}

'Based on Chi-square test without Yates' correction. Statistically significant values are shown in bold.

and providing structural support for cells in addition to influencing cell signaling and polarity. It is also reported that basement membrane breakage is an essential step for the initiation of invasive and metastatic behaviors of most types of cancer (25). In a hamster model of tongue cancer, MMP1 and TIMP1, together with MMP2 and TIMP2, were shown to gradually increase with progression of tongue cancer (26). The invasive and metastatic capacity of oral cancer cells and lymph node metastasis in mouse oral cancer models were all closely related to the expression levels of MMP2 and MMP9 
(27-29). In literature, there are only two articles investigating the contribution of MMP2 genotypes to oral cancer. In 2004, Lin and colleagues reported that the frequency of the $\mathrm{CC}$ genotype at MMP2 -1306 was significantly higher in oral squamous cell carcinoma cases than in controls $(p=0.04)$ (30). In 2006, O-Charoenrat and colleagues further assessed the expression level of MMP2 in serum association in addition to the contribution of $M M P 2$-1306 genotypes to the risk of head and neck cancer (31). They found that the $\mathrm{C}$ and $\mathrm{T}$ allelic frequencies were $93.1 \%$ and $6.9 \%$, respectively, in patients, compared with $87.2 \%$ and $12.8 \%$, respectively, in controls, and the CC genotype frequency was significantly higher in patients than in controls $(86.2 \%$ vs. $76 \%$, $p<0.05)$. Moreover, they found that the expression level of MMP2 in head and neck cancer cells carrying the CC genotype was significantly higher than that in cells carrying the CT genotype. Our findings are consistent with their mentioning that the T-allele of MMP2 -1306 may serve as a protective marker. However, because of their moderate sample numbers (controls: cases $=288 / 242$ and 496/478, respectively) and limited number of studies, this conclusion should be interpreted with caution and further studies, especially those with larger samples, are needed to validate all the findings above.

ECM and MMPs are all important to the etiology of oral cancer. Previously, we investigated the contribution of genomic variants of other MMPs to oral cancer susceptibility among Taiwanese. In 2016, we found that $1 \mathrm{G} / 2 \mathrm{G}$ genotype of $M M P 1$ promoter -1607 had a protective effect on oral cancer risk for smokers (9). Subsequently, we showed that genotypes at MMP8 C-799T, Val436Ala (32) and MMP7 C$153 \mathrm{~T}$ (33) appear not to play a major role in mediating personal risk of oral cancer. The contribution of the genotypes of other MMPs, especially for those whose proteins were proved to be differentially expressed in tumoral and non-tumoral sites, should be examined. In addition, the status of each MMP are also under the control of a complex network at several levels, including through their interactions with specific inhibitors, e.g. the tissue inhibitors of metalloproteinases (TIMPs) (11). Taking MMP2 as an example, the dynamic balance between MMP2 and TIMP2 plays a pivotal role in the maintenance of normal physiological conditions for cells, but it seems that the balance between MMP2 and TIMP2 in oral tissues is not as simple as a 'see-saw' relationship. In the near future, an overall analysis of MMP2 and TIMP2 genotype/phenotype may provide further evidence for evaluating the contribution of these genotypes to oral carcinogenesis.

In conclusion, these results provide evidence showing that the variant $\mathrm{CT}$ and TT genotypes at $M M P 2$ promoter 1306 were protective biomarkers for determining not only susceptibility to oral cancer, but also metastatic behavior in prognosis for Taiwanese.

\section{Acknowledgements}

The Authors declare no conflicts of interest in regard to this study. We express our appreciation to the Tissue-bank of China Medical University Hospital for their excellent sample collection, and $\mathrm{Su}-\mathrm{Yi}$ Pan, Chia-Wen Lin, Cin-Wun Wu, Chia-Yi Pan and Chi-Hsuan Cheng for their technical assistance. This study was supported mainly by the Taiwan Ministry of Science and Technology to Professor Bau (MOST-106-2320-B-039-035) and the China Medical University and Hospital to Professor Bau (DMR-108-124).

\section{References}

1 Torre LA, Bray F, Siegel RL, Ferlay J, Lortet-Tieulent J and Jemal A: Global cancer statistics, 2012. CA Cancer J Clin 65: 87-108, 2015.

2 Taiwan: Cancer Registration System Annual Report. Department of Health, Taiwan, ROC; 2017.

3 Tsai CW, Chang WS, Lin KC, Shih LC, Tsai MH, Hsiao CL, Yang MD, Lin CC and Bau DT: Significant association of Interleukin-10 genotypes and oral cancer susceptibility in Taiwan. Anticancer Res 34: 3731-3737, 2014.

4 Tsai CW, Chang WS, Liu JC, Tsai MH, Lin CC and Bau DT: Contribution of DNA double-strand break repair gene XRCC3 genotypes to oral cancer susceptibility in Taiwan. Anticancer Res 34: 2951-2956, 2014.

5 Tsai CW, Tsai MH, Tsou YA, Shih LC, Tseng HC, Chang WS, Ho CY, Lee HZ and Bau DT: The joint effect of smoking and hOGG1 genotype on oral cancer in Taiwan. Anticancer Res 32: 3799-3803, 2012.

6 Bau DT, Tsai CW, Lin CC, Tsai RY and Tsai MH: Association of alpha B-crystallin genotypes with oral cancer susceptibility, survival, and recurrence in Taiwan. PLoS One 6: e16374, 2011.

7 Tsai CW, Hsu CF, Tsai MH, Tsou YA, Hua CH, Chang WS, Lin $\mathrm{CC}$ and Bau DT: Methylenetetrahydrofolate reductase (MTHFR) genotype, smoking habit, metastasis and oral cancer in Taiwan. Anticancer Res 31: 2395-2399, 2011.

8 Bau DT, Chang CH, Tsai MH, Chiu CF, Tsou YA, Wang RF, Tsai $\mathrm{CW}$ and Tsai RY: Association between DNA repair gene ATM polymorphisms and oral cancer susceptibility. Laryngoscope 120: $2417-2422,2010$

9 Sun KT, Tsai CW, Chang WS, Shih LC, Chen LY, Tsai MH, Ji HX, Hsiao CL, Liu YC, Li CY and Bau DT: The contribution of matrix metalloproteinase-1 genotype to oral cancer susceptibility in Taiwan. In Vivo 30: 439-444, 2016.

10 Woessner JF Jr.: Matrix metalloproteinases and their inhibitors in connective tissue remodeling. FASEB J 5: 2145-2154, 1991.

11 Murphy G and Docherty AJ: The matrix metalloproteinases and their inhibitors. Am J Respir Cell Mol Biol 7: 120-125, 1992.

12 Singh RD, Haridas N, Patel JB, Shah FD, Shukla SN, Shah PM and Patel PS: Matrix metalloproteinases and their inhibitors: correlation with invasion and metastasis in oral cancer. Indian $\mathbf{J}$ Clin Biochem 25: 250-259, 2010.

13 Kesanakurti D, Chetty C, Dinh DH, Gujrati M and Rao JS: Role of MMP-2 in the regulation of IL-6/STAT3 survival signaling via interaction with alpha5beta1 integrin in glioma. Oncogene 32: 327-340, 2013.

14 Ye S: Polymorphism in matrix metalloproteinase gene promoters: implication in regulation of gene expression and susceptibility of various diseases. Matrix Biol 19: 623-629, 2000. 
15 Waleh NS, Murphy BJ and Zaveri NT: Increase in tissue inhibitor of metalloproteinase-2 (TIMP-2) levels and inhibition of MMP-2 activity in a metastatic breast cancer cell line by an anti-invasive small molecule SR13179. Cancer Lett 289: 111$118,2010$.

16 Bourboulia D, Han H, Jensen-Taubman S, Gavil N, Isaac B, Wei B, Neckers L and Stetler-Stevenson WG: TIMP-2 modulates cancer cell transcriptional profile and enhances E-cadherin/betacatenin complex expression in A549 lung cancer cells. Oncotarget 4: 166-176, 2013.

17 Groblewska M, Mroczko B, Kozlowski M, Niklinski J, Laudanski $\mathrm{J}$ and Szmitkowski M: Serum matrix metalloproteinase 2 and tissue inhibitor of matrix metalloproteinases 2 in esophageal cancer patients. Folia Histochem Cytobiol 50: 590-598, 2012.

18 Kapral M, Wawszczyk J, Jurzak M, Dymitruk D and Weglarz L: Evaluation of the expression of metalloproteinases 2 and 9 and their tissue inhibitors in colon cancer cells treated with phytic acid. Acta Pol Pharm 67: 625-629, 2010.

19 Patel BP, Shah PM, Rawal UM, Desai AA, Shah SV, Rawal RM and Patel PS: Activation of MMP-2 and MMP-9 in patients with oral squamous cell carcinoma. J Surg Oncol 90: 81-88, 2005.

20 Srivastava P, Pandey S, Mittal B and Mittal RD: No association of matrix metalloproteinase (MMP)-2 $(-735 \mathrm{C}>\mathrm{T})$ and tissue inhibitor of metalloproteinase (TIMP)-2 $(-418 \mathrm{G}>\mathrm{C})$ gene polymorphisms with cervical cancer susceptibility. Indian J Clin Biochem 28: 13-18, 2013.

21 Nagase H and Woessner JF Jr.: Matrix metalloproteinases. J Biol Chem 274: 21491-21494, 1999.

22 Kessenbrock K, Plaks V and Werb Z: Matrix metalloproteinases: Regulators of the tumor microenvironment. Cell 141: 52-67, 2010.

23 Roy R, Yang J and Moses MA: Matrix metalloproteinases as novel biomarkers and potential therapeutic targets in human cancer. J Clin Oncol 27: 5287-5297, 2009.

24 Bjorklund $\mathrm{M}$ and Koivunen E: Gelatinase-mediated migration and invasion of cancer cells. Biochim Biophys Acta 1755: 37-69, 2005.

25 Mook OR, Frederiks WM and Van Noorden CJ: The role of gelatinases in colorectal cancer progression and metastasis. Biochim Biophys Acta 1705: 69-89, 2004.
26 Fan H, Jiang W, Li H, Fang M, Xu Y and Zheng J: MMP-1/2 and TIMP-1/2 expression levels, and the levels of collagenous and elastic fibers correlate with disease progression in a hamster model of tongue cancer. Oncol Lett 11: 63-68, 2016.

27 Kamide D, Yamashita T, Araki K, Tomifuji M, Tanaka Y, Tanaka S, Shiozawa S and Shiotani A: Selective activator protein-1 inhibitor T-5224 prevents lymph node metastasis in an oral cancer model. Cancer Sci 107: 666-673, 2016.

28 Chung HH, Chen MK, Chang YC, Yang SF, Lin CC and Lin $\mathrm{CW}$ : Inhibitory effects of Leucaena leucocephala on the metastasis and invasion of human oral cancer cells. Environ Toxicol 32: 1765-1774, 2017.

29 Yang JS, Lin $\mathrm{CW}$, Hsin $\mathrm{CH}$, Hsieh $\mathrm{MJ}$ and Chang YC: Selaginella tamariscina attenuates metastasis via AKT pathways in oral cancer cells. PLoS One 8: e68035, 2013.

30 Lin SC, Lo SS, Liu CJ, Chung MY, Huang JW and Chang KW: Functional genotype in matrix metalloproteinases-2 promoter is a risk factor for oral carcinogenesis. J Oral Pathol Med 33: 405409, 2004.

31 O-Charoenrat $\mathrm{P}$ and Khantapura $\mathrm{P}$ : The role of genetic polymorphisms in the promoters of the matrix metalloproteinase2 and tissue inhibitor of metalloproteinase- 2 genes in head and neck cancer. Oral Oncol 42: 257-267, 2006.

32 Hung YW, Tsai CW, Wu CN, Shih LC, Chen YY, Liu YF, Hung HS, Shen MY, Chang WS and Bau DT: The contribution of matrix metalloproteinase-8 promoter polymorphism to oral cancer susceptibility. In Vivo 31: 585-590, 2017.

33 Shih LC, Li CH, Sun KT, Chen LY, Hsu CL, Hung YW, Wu CN, Hsia TC, Shen TC, Chang WS, Shih TC, Tsai CW and Bau DT: Association of matrix metalloproteinase-7 genotypes to the risk of oral cancer in Taiwan. Anticancer Res 38: 2087-2092, 2018.

Received October 24, 2018

Revised October 30, 2018

Accepted November 1, 2018 